\title{
28 Resarch Square \\ Validity of Self-reported Diabetes Among Kurdish Population; a Large Population-based Study From Iran
}

\section{Yousef Moradi}

Kurdistan University of Medical Sciences

Negar Piri

Kurdistan University of Medical Sciences

Hojat Dehghanbanadaki

Tehran University of Medical Sciences

Ghobad Moradi

Kurdistan University of Medical Sciences

Mahdiyeh Fotouk-Kiai

Kurdistan University of Medical Sciences

Farhad Moradpour ( $\square$ farhadmepid@muk.ac.ir)

Kurdistan University of Medical Sciences

\section{Research}

Keywords: Self-report, Diabetes, Validity, Accuracy, Iran

Posted Date: October 29th, 2021

DOl: https://doi.org/10.21203/rs.3.rs-1005210/v1

License: (a) This work is licensed under a Creative Commons Attribution 4.0 International License. Read Full License 


\section{Abstract}

Background: The aim was to assess the validity of diabetes self-reporting based on oral anti-diabetic drugs, insulin injection, or high fasting blood sugar (FBS) in a large Kurdish population of Iran.

Methods: We performed a cross-sectional analytical study on 4400 subjects aged 35-70 years of the Dehgolan Prospective Cohort Study (DehPCS). The reference for having diabetes was oral hypoglycemic drug consumption, insulin injection, or high FBS representing diabetes. Self-reported diabetes status was investigated by well-trained interviewers before the identification of diabetes status based on reference criteria. The accuracy and agreement of self-reported diabetes with reference were assessed in the overall population as well as in different sociodemographic and habitual conditions.

Results: Out of 4400 adults, 3996 agreed to participate in this study (participation=90.8\%). The diabetes prevalence among the study population was $13.1 \%$ based on self-report and $9.7 \%$ based on reference. Of the 523 people who reported diabetes, $213(41.28 \%)$ did not have diabetes. We found a good agreement of $92.3 \%$ concordance with a kappa value of $65.1 \%$ between self-reported diabetes and reference. Self-reported diabetes also guaranteed sensitivity of $78.5 \%$, specificity of $93.9 \%$, positive predictive value of $58.7 \%$, and negative predictive value of $98.0 \%$ to identify diabetic participants.

Conclusion: Self-reported diabetes is identified as a valid tool that could accurately determine the diabetes prevalence in epidemiological studies on the Kurdish population of Iran.

\section{Introduction}

Diabetes is an important risk factor for cardiovascular diseases, a number of cancers, and death, which in recent years has been known as the latent epidemic [1]. The latest data released by the International Diabetes Federation show that there are currently 463 million adults aged 20-79 with diabetes, and $79 \%$ of whom live in low- and middle-income countries [2]. According to the World Health Organization in 2016, the overall prevalence of diabetes in Iran was $10.3 \%$ and, $2 \%$ of the leading causes of death was due to diabetes in 2012 in Iran [3].

There are several ways to evaluate diabetes, of which self-reporting by people is one of the easiest methods [4]. In fact, health assessment through self-reporting in large population-based studies can be an alternative to more complex processes and higher-cost methods for data collection (5). This has led to self-report being used as a valid method to determine the status of diabetes in many different countries such as Japan [1, 5], China [4], the USA [6, 7], and Spain [8]. However, self-reporting may be biased. Respondents may classify themselves as ill when they are not (false positive) or do not report illness if they really are (false negative) [5].

The reference criterion for diagnosing diabetes is a blood sugar test or a history of using blood sugar-lowering drugs. Studies based on patients' self-reporting provide valuable information at a lower cost, but there is still no general agreement on the reliability of such data in different cultures [9]. Different studies have found differences between the results of subjective assessment and standard diagnoses of diabetes [10,11]. In fact, it can be said that the reliability and validity of self-reporting can be related to the various sociodemographic status, as well as the type of chronic diseases [9]. Individuals may not be able to diagnose their condition because they have provided incorrect information to physicians, or have forgotten or misinterpreted medical advice, or even have received incorrect information from specialists [5]. However, studies show that about half of people with diabetes are aware of their disease [2] and only one-fifth of these people have controlled diabetes. So, the accuracy and 
validity of disease estimates could be affected by self-reporting of people [12]. Analyzing self-reported data can help us to better understand the quality of self-reported information. Identifying sociodemographic factors influencing self-report validity can also be important for planning public health policies for more vulnerable groups. In fact, it is important both to interpret existing data and to planning future research on the diagnosis of diabetes and its consequences. Understanding the causes of discrepancies between self-reported diabetes and standard criteria is an important basis for determining the most appropriate approach in future research programs. To this end, we used data from the DehPCS study to assess the validity of self-reported diabetes based on reference criteria, including the history of taking oral anti-diabetic drugs, insulin injection, or high fasting blood sugar (FBS).

\section{Materials And Methods}

\section{Study population}

The present study is a cross-sectional analytical study using enrollment phase data of the Dehgolan Prospective Cohort Study (DehPCS). DehPCS is one of 18 prospective epidemiological cohort studies in Iran (PERSIAN), which is being performed on the population of 35-70 years old, permanent residents of Dehgolan with the aim of assessing the risk factors of common non-communicable diseases in the region. All PERSIAN sites use the same protocol to conduct the study. The questionnaires used in this study have different sections including general factors (demographic, and socioeconomic characteristics, lifestyle, environmental exposure, occupational exposure, physical activity, and personal habits) medical factors (medical history, clinical symptoms, family medical history, drug use, reproductive history, oral health, general health, anthropometry, physical exam, blood and urine analysis) and nutritional factors (food frequency, eating habits, and supplementation). Sampling was done by a simple cluster sampling method. 4400 people were invited to participate in the study. The participation rate was $90.8 \%$ among eligible individuals. Out of a total of 3996 participants, 3976 had adequate information about diabetes self-reporting, blood samples, and taking medication or insulin injection and were considered for further assessment. The study design and rationale for conducting the study were published previously $[13,14]$.

\section{Data collection and measurements}

In the first step, participants were invited to the study site. Initially, the informed consent form was signed by participants. Then, to collect information, they were enrolled in the online software and received a unique code. All data were collected by expert interviewers who had completed the necessary training courses according to the executive protocol. For para-clinical tests, biological samples (blood and urine) were first collected on an empty stomach. We measured the weight using the Seka scale and the height using the Seka stadiometer to the nearest $0.1 \mathrm{~cm}$. Body mass index (BMI) was calculated as weight in kilograms divided by height in square meters. Blood pressure was measured using a Richter aneroid sphygmomanometer after at least 15 minutes of rest, with two measurements in the right arm at intervals of at least half an hour. The mean of the two measurements was considered as the mean of systolic and diastolic blood pressure. According to the JNC-7 criteria, people with systolic blood pressure $\geq 140 \mathrm{mmHg}$, or diastolic blood pressure $\geq 90 \mathrm{mmHg}$, or people with a history of taking antihypertensive drugs were considered hypertensive. The official age of the participants was considered based on their identity cards. Education was measured based on the number of years the person had studied. Economic status was calculated based on the wealth index using the Multiple Correspondence Analysis (MCA) method with analysis of principal components regarding durable goods, housing features, and other facilities. Individuals with a history of smoking less than 100 cigarettes during their lifetime were considered non-smokers. The use of illicit drugs was defined as the use of drugs once a week for at least six months, and alcohol consumption as drinking about $200 \mathrm{ml}$ of beer or $45 \mathrm{ml}$ of alcohol once a week for at least six months. Family history of diabetes was also 
assessed in first- and second-degree relatives. Second-degree relatives refer to people with whom we share $25 \%$ of the genome. It is noteworthy that we collected self-reporting diabetes data before identification of diabetes status based on reference criteria.

\section{Diabetes measurement}

Diabetes self-reporting was assessed by asking the following question, "Have you ever had diabetes in the past?" People who answered yes, were asked the next question, "Who told you that you had diabetes?" All those who answered diagnosed by a physician were considered to have self-reported diabetes. The reference criterion for the diagnosis of diabetes included abnormal fasting blood sugar (FBS) indicating diabetes or positive history of routine insulin use or oral hypoglycemic drugs. FBS $\geq 126 \mathrm{mg} / \mathrm{dL}$ ( $7 \mathrm{mmol} / \mathrm{L}$ ) was considered diabetes. Drug use on the day of blood sampling was assessed with the following question, "Do you routinely use anti-diabetic drugs or insulin?" If the answer was yes, the used drugs were visually evaluated.

\section{Statistical analysis}

Diabetes self-reporting validation was performed using the following criteria. Sensitivity (Se) as a proportion of people with DM who had self-reported diabetes (true positive/[true positive + false negative]), Specificity (Se) as a proportion of people without DM who did not have self-reported diabetes or true negative rate (true negative/[true negative + false positive], Positive Predictive Value (PPV) as a proportion of individuals with self-reported DM who had the reference-based DM (true positive/[true positive + false positive], Negative Predictive Value (NPV) as a proportion of individuals without self-reported DM who did not have reference-based DM (true negative/[true negative + false negative], Positive Likelihood Ratio (LR+) as Se divided by false positive rate (FPR), Negative Likelihood Ratio (LR-) as false negative rate (FNR) divided by Sp. Kappa coefficient was another calculated statistics. kappa examines free chance concordance between two diagnostic approaches. 95\% Confidence Intervals $(\mathrm{Cl})$ were calculated for all values based on the standard method for proportion. Validity was calculated overall and based on demographic, and socioeconomic characteristics, three categories of body mass index (BMI), personal habits, and hypertension status. Binary and multinomial logistic regression was used to examine concordance between self-reported diabetes and the reference value. To examine diagnostic characteristics of selfreported diabetes plus sex and age, we used Precision-Recall Curve (PRC). PRC presents PPV against Se. The twosided test with an alpha level of 0.05 was considered for statistical significance. All analysis was done by using Stata software version 16 (Stata Corp, College Station, TX, USA).

\section{Results}

Out of 3976 participants with adequate information about diabetes self-reporting and their reference criteria, 2241 (56.26\%) participants were female and 1735 (43.74\%) were male. The mean age of male and female participants was $47.98 \pm 8.91$ years and $48.78 \pm 8.91$ years, respectively. Most participants had a lower level of education than high school, and about $31 \%$ of them were illiterate. The mean BMI of the participants was $28.00 \pm 4.58 \mathrm{~kg} / \mathrm{m}^{2} \mathrm{and}$ $32.31 \%$ of them were in the obese group with $\mathrm{BMI} \geq 30 \mathrm{~kg} / \mathrm{m}^{2}$. In terms of blood pressure, $21.50 \%$ of them had a systolic blood pressure $\geq 140$ or a diastolic blood pressure $\geq 90$. Also, $27.81 \%$ of people reported a history of diabetes in their first-degree relatives. Demographic characteristics and basic information of the participants were shown in Table 1. 
Table 1

demographic characteristic and baseline information of the participants in DehPCS, by self-reported diabetes situation

\begin{tabular}{|c|c|c|c|c|}
\hline & Total $\mathbf{n}$ & Diabetic n (\%) & Non-diabetic n (\%) & P-value \\
\hline \multicolumn{5}{|l|}{ Gender } \\
\hline Male & 1,735 & $151(8.70)$ & $1,584(91.30)$ & \multirow[t]{2}{*}{$<0.001$} \\
\hline Female & 2,241 & $372(16.60)$ & $1,869(83.40)$ & \\
\hline \multicolumn{5}{|l|}{ Age groups } \\
\hline $35-45$ & 1,773 & $149(8.40)$ & $1,624(91.60)$ & \multirow[t]{3}{*}{$<0.001$} \\
\hline $46-60$ & 1,720 & $261(15.17)$ & $1,459(84.83)$ & \\
\hline$>60$ & 483 & $113(23.40)$ & $370(76.60)$ & \\
\hline \multicolumn{5}{|l|}{ Marital status } \\
\hline Married & 3651 & $453(12.41)$ & 3198 (87.59) & \multirow[t]{2}{*}{$<0.001$} \\
\hline Single & 325 & $70(21.54)$ & $255(78.46)$ & \\
\hline \multicolumn{5}{|l|}{ Education years } \\
\hline Illiterate & 1,245 & $262(21.04)$ & 983 (78.96) & \multirow[t]{4}{*}{$<0.001$} \\
\hline $1-5$ & 1,110 & $142(12.79)$ & $968(87.21)$ & \\
\hline $6-12$ & 1,113 & $78(7.01)$ & 1,035 (92.99) & \\
\hline university & 508 & $41(8.07)$ & 467 (91.93) & \\
\hline \multicolumn{5}{|c|}{ Economic status } \\
\hline Poorest & 1,344 & 149 (11.09) & 1,195 (88.91) & \multirow[t]{3}{*}{0.001} \\
\hline Moderate & 1,300 & 165 (12.69) & 1,135 (87.31) & \\
\hline Reach & 1,318 & $208(15.78)$ & $1,110(84.22)$ & \\
\hline \multicolumn{5}{|l|}{ BMI } \\
\hline Normal weight & 985 & $75(7.61)$ & 910 (92.39) & \multirow[t]{3}{*}{$<0.001$} \\
\hline Over-weight & 1698 & $246(14.49)$ & $1452(85.51)$ & \\
\hline Obese & 1281 & 201 (15.69) & $1080(84.31)$ & \\
\hline \multicolumn{5}{|c|}{ Cigarette smoking } \\
\hline No smoker & 3024 & 393 (13.00) & $2631(87.00)$ & \multirow[t]{3}{*}{$<0.001$} \\
\hline Ex-smoker & 326 & $68(20.86)$ & $258(79.14)$ & \\
\hline Smoker & 600 & $61(10.17)$ & 539 (89.83) & \\
\hline \multicolumn{5}{|l|}{ Drug use } \\
\hline Yes & 448 & $47(10.49)$ & 401 (89.51) & 0.071 \\
\hline
\end{tabular}




\begin{tabular}{|c|c|c|c|c|}
\hline & Total $\mathbf{n}$ & Diabetic n (\%) & Non-diabetic n (\%) & P-value \\
\hline No & 3502 & 475 (13.56) & 3027 (86.44) & \\
\hline \multicolumn{5}{|l|}{ Alcohol use } \\
\hline Yes & 481 & $45(9.36)$ & $436(90.64)$ & \multirow[t]{2}{*}{0.008} \\
\hline No & 3470 & $477(13.75)$ & $2993(86.25)$ & \\
\hline \multicolumn{5}{|l|}{ HTN } \\
\hline Yes & 839 & $182(21.69)$ & $657(78.31)$ & \multirow[t]{2}{*}{$<0.001$} \\
\hline No & 3137 & $341(10.87)$ & $2796(89.13)$ & \\
\hline \multicolumn{5}{|c|}{ Family history of DM } \\
\hline No & 2436 & $234(9.61)$ & $2202(90.39)$ & \multirow[t]{3}{*}{$<0.001$} \\
\hline Second degree & 430 & $63(14.65)$ & 367 (85.35) & \\
\hline First degree & 1106 & $226(20.43)$ & $880(79.57)$ & \\
\hline
\end{tabular}

The prevalence of diabetes based on self-report was $13.1 \%$, and this estimate based on reference criteria was $9.7 \%$ among the study population. Of the 523 people who reported diabetes, $213(41.28 \%)$ did not have diabetes according to the reference criteria. Among people treated for diabetes, 167 (63.74\%) people had poorly controlled diabetes, and among people with diabetes (with high FBS or treated with drugs or insulin (21.50\%)), 83 did not know they had it (Figure 1).

Table 2 shows the validation of diabetes self-reporting based on demographic, socioeconomic, and some individual variables. The percentage of general agreement and agreement based on kappa statistics was $92.3 \%$ and $65.1 \%$, respectively. The estimated value of kappa statistics varied between $45.5 \%$ and $81.1 \%$ based on the characteristics of the individuals under study. In general, the kappa agreement was higher in men, older age groups, people with poor economic status, and people with normal weight, ex-smokers, and people with high blood pressure. The overall Se and Sp were $78.5 \%$ and $93.9 \%$, respectively. Se increased and decreased with age and weight, respectively. The total PPV and NPV were $58.7 \%$ and $98.0 \%$, respectively. Unlike Se, PPV was significantly higher in men than in women. With age, PPV increased by more than $38 \%$ so that in the age group over 60 years, it reached more than $72 \%$. Also, PPV was higher among people with hypertension and people with a family history of diabetes. Figure 2 shows the PRC. The area under the curve was $64.24 \%$ for the full model and $61.60 \%$ for the reduced model. 
Table 2

validity of self-reported diabetes using reference criteria in DehPCS

\begin{tabular}{|c|c|c|c|c|c|c|c|c|}
\hline & $\begin{array}{l}\text { Concordance\% } \\
\text { (Cl) }\end{array}$ & $\begin{array}{l}\text { Kappa\% } \\
\text { (CI) }\end{array}$ & $\begin{array}{l}\text { Sensitivity\% } \\
\text { (Cl) }\end{array}$ & $\begin{array}{l}\text { Specificity\% } \\
\text { (Cl) }\end{array}$ & $+\mathrm{LR}$ & -LR & $\begin{array}{l}\text { PPV\% } \\
\text { (Cl) }\end{array}$ & $\begin{array}{l}\text { NPV\% } \\
\text { (Cl) }\end{array}$ \\
\hline Overall & $\begin{array}{l}92.3(90.9- \\
93.7)\end{array}$ & $\begin{array}{l}65.1 \\
(61.8- \\
68.3)\end{array}$ & $\begin{array}{l}78.5(74.1- \\
82.5)\end{array}$ & $\begin{array}{l}93.9(93.0- \\
94.6)\end{array}$ & $\begin{array}{l}12.78 \\
(11.11- \\
14.70)\end{array}$ & $\begin{array}{l}0.23 \\
(0.19- \\
0.28)\end{array}$ & $\begin{array}{l}58.7 \\
(54.3- \\
63.0)\end{array}$ & $\begin{array}{l}98.0 \\
(97.4- \\
98.4)\end{array}$ \\
\hline \multicolumn{9}{|l|}{ Gender } \\
\hline Female & $\begin{array}{l}90.2(88.3- \\
92.1)\end{array}$ & $\begin{array}{l}61.6 \\
(57.3- \\
65.9)\end{array}$ & $\begin{array}{l}84.2(78.9- \\
88.6)\end{array}$ & $\begin{array}{l}91.3(90.0- \\
92.5)\end{array}$ & $\begin{array}{l}9.71 \\
(8.34- \\
11.31)\end{array}$ & $\begin{array}{l}0.17 \\
(0.13- \\
0.23)\end{array}$ & $\begin{array}{l}53.8 \\
(48.7- \\
59.0)\end{array}$ & $\begin{array}{l}98.0 \\
(97.3- \\
98.6)\end{array}$ \\
\hline Male & $\begin{array}{l}95.1(93.0- \\
97.2)\end{array}$ & $\begin{array}{l}72.2 \\
(66.2- \\
76.3)\end{array}$ & $\begin{array}{l}76.1(68.1- \\
82.9)^{(1)}\end{array}$ & $\begin{array}{l}97.1(96.2- \\
97.9)\end{array}$ & $\begin{array}{l}26.42 \\
(19.57- \\
36.65)\end{array}$ & $\begin{array}{l}0.25 \\
(0.18- \\
0.33)\end{array}$ & $\begin{array}{l}70.7 \\
(62.7- \\
77.8)\end{array}$ & $\begin{array}{l}97.3 \\
(96.4- \\
98.1)\end{array}$ \\
\hline \multicolumn{9}{|l|}{$\begin{array}{l}\text { Age } \\
\text { groups }\end{array}$} \\
\hline $35-45$ & $\begin{array}{l}93.2(91.0- \\
95.2)\end{array}$ & $\begin{array}{l}45.5 \\
(40.8- \\
50.2)\end{array}$ & $\begin{array}{l}69.9(58.0- \\
80.1)\end{array}$ & $\begin{array}{l}94.2(93.0- \\
95.3)\end{array}$ & $\begin{array}{l}12.12 \\
(9.49- \\
15.47)\end{array}$ & $\begin{array}{l}0.32 \\
(0.23- \\
0.45)\end{array}$ & $\begin{array}{l}34.2 \\
(26.7- \\
42.4)\end{array}$ & $\begin{array}{l}98.6 \\
(98.0- \\
99.1)\end{array}$ \\
\hline $46-60$ & $\begin{array}{l}92.5(90.3- \\
94.7)\end{array}$ & $\begin{array}{l}70.8 \\
(65.8- \\
75.8)\end{array}$ & $\begin{array}{l}85.0(79.3- \\
89.6)\end{array}$ & $\begin{array}{l}94.0(92.7- \\
95.2)\end{array}$ & $\begin{array}{l}14.20 \\
(11.54- \\
17.47)\end{array}$ & $\begin{array}{l}0.16 \\
(0.11- \\
0.22)\end{array}$ & $\begin{array}{l}65.1 \\
(59.0- \\
70.9)\end{array}$ & $\begin{array}{l}97.9 \\
(97.1- \\
98.6)\end{array}$ \\
\hline$>60$ & $\begin{array}{l}88.7(84.5- \\
92.9)\end{array}$ & $\begin{array}{l}70.5 \\
(61.6- \\
79.4)\end{array}$ & $\begin{array}{l}82.0(73.1- \\
89.0)\end{array}$ & $\begin{array}{l}91.9(88.7- \\
94.4)\end{array}$ & $\begin{array}{l}10.13 \\
(7.14- \\
14.37)\end{array}$ & $\begin{array}{l}0.20 \\
(0.13- \\
0.30)\end{array}$ & $\begin{array}{l}72.6 \\
(63.4- \\
80.5)\end{array}$ & $\begin{array}{l}95.1 \\
(92.4- \\
97.1)\end{array}$ \\
\hline \multicolumn{9}{|l|}{$\begin{array}{l}\text { Marital } \\
\text { status }\end{array}$} \\
\hline Married & $\begin{array}{l}92.9(91.4- \\
94.3)\end{array}$ & $\begin{array}{l}66.2 \\
(62.8- \\
69.7)\end{array}$ & $\begin{array}{l}81.4(76.8- \\
85.5)\end{array}$ & $\begin{array}{l}94.4(93.6- \\
95.2)\end{array}$ & $\begin{array}{l}14.54 \\
(12.53- \\
16.88)\end{array}$ & $\begin{array}{l}0.20 \\
(0.16- \\
0.25)\end{array}$ & $\begin{array}{l}58.9 \\
(54.3- \\
63.5)\end{array}$ & $\begin{array}{l}98.1 \\
(97.6- \\
98.5)\end{array}$ \\
\hline Single & $\begin{array}{l}\text { 85.9(80.4- } \\
91.3)\end{array}$ & $\begin{array}{l}55.3 \\
(51.9- \\
58.7)\end{array}$ & $\begin{array}{l}79.5 \text { (64.7- } \\
90.2)\end{array}$ & $\begin{array}{l}87.5(83.1- \\
91.2)\end{array}$ & $\begin{array}{l}6.39 \\
(4.53- \\
9.01)\end{array}$ & $\begin{array}{l}0.23 \\
(0.13- \\
0.42)\end{array}$ & $\begin{array}{l}50.0 \\
(37.8- \\
62.2)\end{array}$ & $\begin{array}{l}96.5 \\
(93.4- \\
98.4)\end{array}$ \\
\hline \multicolumn{9}{|l|}{$\begin{array}{l}\text { Education } \\
\text { years }\end{array}$} \\
\hline Illiterate & $\begin{array}{l}89.2(86.6- \\
91.8)\end{array}$ & $\begin{array}{l}66.4 \\
(60.6- \\
72.2)\end{array}$ & $\begin{array}{l}86.4(80.6- \\
91.0)\end{array}$ & $\begin{array}{l}90.3(88.4- \\
92.0)\end{array}$ & $\begin{array}{l}8.90 \\
(7.34- \\
10.79)\end{array}$ & $\begin{array}{l}0.15 \\
(0.10- \\
0.22)\end{array}$ & $\begin{array}{l}60.7 \\
(54.5- \\
66.6)\end{array}$ & $\begin{array}{l}97.5 \\
(96.3- \\
98.3)\end{array}$ \\
\hline $1-5$ & $\begin{array}{l}91.9(89.2- \\
94.6)\end{array}$ & $\begin{array}{l}61.6 \\
(55.4- \\
67.8)\end{array}$ & $\begin{array}{l}79.2(69.7- \\
86.8)\end{array}$ & $\begin{array}{l}93.5(91.8- \\
94.9)\end{array}$ & $\begin{array}{l}12.16 \\
(9.43- \\
15.69)\end{array}$ & $\begin{array}{l}0.22 \\
(0.15- \\
0.33)\end{array}$ & $\begin{array}{l}53.5 \\
(45.0- \\
61.9)\end{array}$ & $\begin{array}{l}97.9 \\
(96.8- \\
98.7)\end{array}$ \\
\hline $6-12$ & $\begin{array}{l}94.9(92.3- \\
97.5)\end{array}$ & $\begin{array}{l}51.5(55.2- \\
67.8)\end{array}$ & $\begin{array}{l}69.8(57.0- \\
80.8)\end{array}$ & $\begin{array}{l}96.8(95.5- \\
97.7)\end{array}$ & $\begin{array}{l}21.57 \\
(14.92- \\
31.17)\end{array}$ & $\begin{array}{l}0.31 \\
(0.21- \\
0.45)\end{array}$ & $\begin{array}{l}56.4 \\
(44.7- \\
67.6)\end{array}$ & $\begin{array}{l}98.2 \\
(97.1- \\
98.9)\end{array}$ \\
\hline university & $\begin{array}{l}95.1(91.4- \\
98.8)\end{array}$ & $\begin{array}{l}65.1 \\
(55.9- \\
74.2)\end{array}$ & $\begin{array}{l}79.3(60.3- \\
92.0)\end{array}$ & $\begin{array}{l}96.2(94.1- \\
97.8)\end{array}$ & $\begin{array}{l}21.11 \\
(12.93- \\
34.45)\end{array}$ & $\begin{array}{l}0.21 \\
(0.11- \\
0.44)\end{array}$ & $\begin{array}{l}56.1 \\
(39.7- \\
71.5)\end{array}$ & $\begin{array}{l}98.7 \\
(97.2- \\
99.5)\end{array}$ \\
\hline
\end{tabular}




\section{$\begin{array}{llllllll}\begin{array}{l}\text { Concordance\% } \\ \text { (Cl) }\end{array} & \begin{array}{l}\text { Kappa\% } \\ \text { (Cl) }\end{array} & \begin{array}{l}\text { Sensitivity\% } \\ \text { (Cl) }\end{array} & \begin{array}{l}\text { Specificity\% } \\ \text { (Cl) }\end{array} & \text { +LR } & \text {-LR } & \begin{array}{l}\text { PPV\% } \\ \text { (Cl) }\end{array} & \begin{array}{l}\text { NPV\% } \\ \text { (Cl) }\end{array}\end{array}$}

Economic

status

\begin{tabular}{|c|c|c|c|c|c|c|c|c|}
\hline Poor & $\begin{array}{l}94.7(92.4- \\
97.0)\end{array}$ & $\begin{array}{l}72.3 \\
(66.7- \\
78.0)\end{array}$ & $\begin{array}{l}87.9(80.1- \\
93.4)\end{array}$ & $\begin{array}{l}95.6(94.3- \\
96.6)\end{array}$ & $\begin{array}{l}19.76 \\
(15.12- \\
25.83)\end{array}$ & $\begin{array}{l}0.13 \\
(0.08- \\
0.21)\end{array}$ & $\begin{array}{l}63.1 \\
\text { (54.8- } \\
70.8)\end{array}$ & $\begin{array}{l}98.9 \\
(98.1- \\
99.4)\end{array}$ \\
\hline Moderate & $\begin{array}{l}91.3(88.7- \\
94.0)\end{array}$ & $\begin{array}{l}60.4 \\
(54.6- \\
66.2)\end{array}$ & $\begin{array}{l}75.6(66.9- \\
83.0)\end{array}$ & $\begin{array}{l}93.6(92.1- \\
95.0)\end{array}$ & $\begin{array}{l}11.91 \\
(9.35- \\
15.16)\end{array}$ & $\begin{array}{l}0.26 \\
(0.19- \\
0.36)\end{array}$ & $\begin{array}{l}54.5 \\
(46.6- \\
62.3)\end{array}$ & $\begin{array}{l}97.4 \\
(96.4- \\
98.3)\end{array}$ \\
\hline Rich & $\begin{array}{l}90.7(88.3- \\
93.1)\end{array}$ & $\begin{array}{l}62.9 \\
(57.3- \\
68.6)\end{array}$ & $\begin{array}{l}80.7 \text { (73.3- } \\
86.8)\end{array}$ & $\begin{array}{l}92.2(90.6- \\
93.7)\end{array}$ & $\begin{array}{l}10.40 \\
(8.41- \\
12.87)\end{array}$ & $\begin{array}{l}0.21 \\
(0.15- \\
0.29)\end{array}$ & $\begin{array}{l}56.3 \\
(49.2- \\
63.1)\end{array}$ & $\begin{array}{l}97.5 \\
(96.4- \\
98.3)\end{array}$ \\
\hline
\end{tabular}

$\mathrm{BMI}$

\begin{tabular}{|c|c|c|c|c|c|c|c|c|}
\hline $\begin{array}{l}\text { Normal } \\
\text { weight }\end{array}$ & $\begin{array}{l}95.9(93.1- \\
98.6)\end{array}$ & $\begin{array}{l}70.3 \\
(63.6- \\
76.9)\end{array}$ & $\begin{array}{l}85.2(72.9- \\
93.4)\end{array}$ & $\begin{array}{l}96.9(95.6- \\
97.9)\end{array}$ & $\begin{array}{l}27.35 \\
(18.79- \\
39.79)\end{array}$ & $\begin{array}{l}0.15 \\
(0.08- \\
0.29)\end{array}$ & $\begin{array}{l}61.3 \\
(49.4- \\
72.4)\end{array}$ & $\begin{array}{l}99.1 \\
(98.3- \\
99.6)\end{array}$ \\
\hline $\begin{array}{l}\text { Over- } \\
\text { weight }\end{array}$ & $\begin{array}{l}92.2(90.1- \\
94.4)\end{array}$ & $\begin{array}{l}68.5 \\
(63.5- \\
73.5)\end{array}$ & $\begin{array}{l}82.9 \text { (76.7- } \\
88.0)\end{array}$ & $\begin{array}{l}94.0(92.7- \\
95.1)\end{array}$ & $\begin{array}{l}13.76 \\
(11.16 \\
16.97)\end{array}$ & $\begin{array}{l}0.18 \\
(0.13- \\
0.25)\end{array}$ & $\begin{array}{l}63.0 \\
(56.6- \\
69.1)\end{array}$ & $\begin{array}{l}97.8 \\
(96.9- \\
98.5)\end{array}$ \\
\hline Obese & $\begin{array}{l}89.7(87.2- \\
92.3)\end{array}$ & $\begin{array}{l}57.8 \\
(52.1- \\
63.5)\end{array}$ & $\begin{array}{l}77.7(69.6- \\
84.5)\end{array}$ & $\begin{array}{l}91.3(89.5- \\
92.9)\end{array}$ & $\begin{array}{l}8.94 \\
(7.26- \\
11.02)\end{array}$ & $\begin{array}{l}0.24 \\
(0.18 \\
-0.34)\end{array}$ & $\begin{array}{l}50.2 \\
(43.1- \\
57.4)\end{array}$ & $\begin{array}{l}97.3 \\
(96.2- \\
98.2)\end{array}$ \\
\hline
\end{tabular}

Cigarette

smoking

\begin{tabular}{|c|c|c|c|c|c|c|c|c|}
\hline $\begin{array}{l}\text { No } \\
\text { smoker }\end{array}$ & $\begin{array}{l}92.0(90.4- \\
93.6)\end{array}$ & $\begin{array}{l}62.3 \\
(58.5- \\
66.0)\end{array}$ & $\begin{array}{l}80.6 \text { (75.3- } \\
85.2)\end{array}$ & $\begin{array}{l}93.4(92.5- \\
94.3)\end{array}$ & $\begin{array}{l}12.30 \\
(10.55- \\
14.33)\end{array}$ & $\begin{array}{l}0.21 \\
(0.16- \\
0.27)\end{array}$ & $\begin{array}{l}53.9 \\
(48.9- \\
59.0)\end{array}$ & $\begin{array}{l}98.1 \\
(97.5- \\
98.6)\end{array}$ \\
\hline $\begin{array}{l}\text { Ex- } \\
\text { smoker }\end{array}$ & $\begin{array}{l}93.7(89.1- \\
98.4)\end{array}$ & $\begin{array}{l}81.1 \\
(69.6- \\
92.6)\end{array}$ & $\begin{array}{l}94.4(84.6- \\
98.8)\end{array}$ & $\begin{array}{l}93.8(90.2- \\
96.3)\end{array}$ & $\begin{array}{l}15.11 \\
(9.49- \\
24.05)\end{array}$ & $\begin{array}{l}0.06 \\
(0.02- \\
0.18)\end{array}$ & $\begin{array}{l}75.0 \\
(63.0- \\
84.7)\end{array}$ & $\begin{array}{l}98.8 \\
(96.6- \\
99.8)\end{array}$ \\
\hline Smoker & $\begin{array}{l}92.9(89.4- \\
96.5)\end{array}$ & $\begin{array}{l}63.3 \\
\text { (54.8- } \\
71.9)\end{array}$ & $\begin{array}{l}70.4(56.4- \\
82.0)\end{array}$ & $\begin{array}{l}95.8(93.7- \\
97.3)\end{array}$ & $\begin{array}{l}16.71 \\
(10.80- \\
25.83)\end{array}$ & $\begin{array}{l}0.31 \\
(0.20- \\
0.47)\end{array}$ & $\begin{array}{l}62.3 \\
(49.0- \\
74.4)\end{array}$ & $\begin{array}{l}97.0 \\
(95.2- \\
98.3)\end{array}$ \\
\hline
\end{tabular}

Drug use

$\begin{array}{lllllllll}\text { No } & 92.0(90.5- & 64.4 & 81.6(77.0- & 93.5(92.6- & 12.62 & 0.20 & 56.8 & 98.0 \\ & 93.5) & (60.9- & 85.6) & 94.4) & (10.95- & (0.16- & (52.3- & (97.4- \\ & & 67.9) & & & 14.54) & 0.25) & 61.3) & 98.5) \\ \text { Yes } & 94.2(90.3- & 69.7 & 77.5(61.5- & 96.1(93.7- & 19.76 & 0.23 & 66.0 & 97.8 \\ & 98.1) & (59.9- & 89.2) & 97.7) & (11.89- & (0.13- & (50.7- & (95.8- \\ & & 79.5) & & & 32.86) & 0.42) & 79.1) & 99.0)\end{array}$

Alcohol

use

No

$92.0(90.5$
$93.5)$

65.2
$(61.7-$
$68.7)$

$\begin{array}{ll}81.3(76.7- & 93.6(92.7- \\ 85.3) & 94.5)\end{array}$

$\begin{array}{llll}12.78 & 0.20 & 58.3 & 97.9 \\ (11.07- & (0.16- & (53.7- & (97.3- \\ 14.75) & 0.25) & 62.7) & 98.3)\end{array}$ 


\begin{tabular}{|c|c|c|c|c|c|c|c|c|}
\hline & $\begin{array}{l}\text { Concordance\% } \\
\text { (Cl) }\end{array}$ & $\begin{array}{l}\text { Kappa\% } \\
\text { (CI) }\end{array}$ & $\begin{array}{l}\text { Sensitivity\% } \\
\text { (Cl) }\end{array}$ & $\begin{array}{l}\text { Specificity\% } \\
\text { (Cl) }\end{array}$ & +LR & -LR & $\begin{array}{l}\text { PPV\% } \\
\text { (Cl) }\end{array}$ & $\begin{array}{l}\text { NPV\% } \\
\text { (Cl) }\end{array}$ \\
\hline Yes & $\begin{array}{l}94.0(90.1- \\
98.0)\end{array}$ & $\begin{array}{l}61.7 \\
(52.3- \\
71.0)\end{array}$ & $\begin{array}{l}79.3 \text { (60.3- } \\
92.0)\end{array}$ & $\begin{array}{l}95.1 \text { (92.7- } \\
96.9)\end{array}$ & $\begin{array}{l}16.29 \\
(10.41- \\
25.50)\end{array}$ & $\begin{array}{l}0.22 \\
(0.11- \\
0.44)\end{array}$ & $\begin{array}{l}51.1 \\
(35.8- \\
66.3)\end{array}$ & $\begin{array}{l}98.6 \\
(97.0- \\
99.5)\end{array}$ \\
\hline \multicolumn{9}{|l|}{ HTN } \\
\hline No & $\begin{array}{l}92.7(91.2- \\
94.3)\end{array}$ & $\begin{array}{l}59.6 \\
(56.0- \\
63.3)\end{array}$ & $\begin{array}{l}77.9)^{71.9-} \\
83.2(\end{array}$ & $\begin{array}{l}94.2 \text { (93.3- } \\
95.1)\end{array}$ & $\begin{array}{l}13.52 \\
(11.49- \\
15.91)\end{array}$ & $\begin{array}{l}0.23 \\
(0.18- \\
0.30)\end{array}$ & $\begin{array}{l}50.7 \\
(45.3- \\
56.2)\end{array}$ & $\begin{array}{l}98.2 \\
(97.7- \\
98.7)\end{array}$ \\
\hline Yes & $\begin{array}{l}90.7 \text { (87.4- } \\
93.9)\end{array}$ & $\begin{array}{l}73.2 \\
(65.9- \\
80.4)\end{array}$ & $\begin{array}{l}86.0(79.4- \\
91.1)\end{array}$ & $\begin{array}{l}92.3(90.1- \\
94.2)\end{array}$ & $\begin{array}{l}11.18 \\
(8.56- \\
14.60)\end{array}$ & $\begin{array}{l}0.15 \\
(0.10- \\
0.23)\end{array}$ & $\begin{array}{l}70.9 \\
(63.7- \\
77.4)\end{array}$ & $\begin{array}{l}96.8 \\
(95.2- \\
98.0)\end{array}$ \\
\hline \multicolumn{9}{|c|}{$\begin{array}{l}\text { Family } \\
\text { history of } \\
\text { DM }\end{array}$} \\
\hline No & $\begin{array}{l}93.0(91.2- \\
94.8)\end{array}$ & $\begin{array}{l}59.4 \\
(55.2- \\
63.6)\end{array}$ & $\begin{array}{l}72.8(65.6- \\
79.3)\end{array}$ & $\begin{array}{l}95.2(94.3- \\
96.1)\end{array}$ & $\begin{array}{l}15.26 \\
(12.43- \\
18.74)\end{array}$ & $\begin{array}{l}0.29 \\
(0.22- \\
0.36)\end{array}$ & $\begin{array}{l}53.8 \\
(47.2- \\
60.4)\end{array}$ & $\begin{array}{l}97.9 \\
(97.2- \\
98.4)\end{array}$ \\
\hline $\begin{array}{l}\text { Second } \\
\text { degree }\end{array}$ & $\begin{array}{l}94.1(90.0- \\
98.3)\end{array}$ & $\begin{array}{l}74.3 \\
(64.4- \\
84.2)\end{array}$ & $\begin{array}{l}95.0(83.1- \\
99.4)\end{array}$ & $\begin{array}{l}93.6(90.7- \\
95.8)\end{array}$ & $\begin{array}{l}14.82 \\
(10.08- \\
21.80)\end{array}$ & $\begin{array}{l}0.05 \\
(0.01- \\
0.21)\end{array}$ & $\begin{array}{l}60.3 \\
(47.2- \\
72.4)\end{array}$ & $\begin{array}{l}99.5 \\
(98.0- \\
99.9)\end{array}$ \\
\hline $\begin{array}{l}\text { First } \\
\text { degree }\end{array}$ & $\begin{array}{l}90.0(87.3- \\
92.7)\end{array}$ & $\begin{array}{l}67.8 \\
(61.6- \\
73.9)\end{array}$ & $\begin{array}{l}86.8(80.5- \\
91.6)\end{array}$ & $\begin{array}{l}90.7(88.7- \\
92.5)\end{array}$ & $\begin{array}{l}9.34 \\
(7.59- \\
11.50)\end{array}$ & $\begin{array}{l}0.15 \\
(0.10- \\
0.22)\end{array}$ & $\begin{array}{l}61.1 \\
(54.4- \\
67.5)\end{array}$ & $\begin{array}{l}97.6 \\
(96.4- \\
98.5)\end{array}$ \\
\hline
\end{tabular}

According to Table 3, in multivariate analysis, independent factors influencing the increase in discrepancy between diabetes self-reporting and the reference value included female gender, celibacy, moderate to high economic status, higher BMI, and having a first-degree relative with diabetes. Polynomial regression results suggested that men, single people, those in the upper economic class, people with higher BMI, and those having a first-degree relative with diabetes were more likely to falsely report diabetes (FP). Conversely, the probability of false reports of not having diabetes was higher in men, older people, and those in the lower economic class. However, female gender, older ages, higher BMI, previous history of smoking, high blood pressure, and family history of diabetes significantly increased the true reports of diabetes mellitus (TP) compared to those of non-diabetes (TN). 
Table 3

univariable and multivariable analysis of factors affecting validity of self-reported diabetes in DehPCS.

\begin{tabular}{|c|c|c|c|c|c|c|c|c|}
\hline \multirow[t]{2}{*}{ Variables } & \multicolumn{2}{|c|}{$\begin{array}{l}\text { disagreement self- } \\
\text { reported }^{1}\end{array}$} & \multicolumn{2}{|c|}{$\begin{array}{l}\text { false positive self- } \\
\text { reported }^{2}\end{array}$} & \multicolumn{2}{|c|}{$\begin{array}{l}\text { false negative self- } \\
\text { reported }^{2}\end{array}$} & \multicolumn{2}{|c|}{$\begin{array}{l}\text { true positive self- } \\
\text { reported }^{2}\end{array}$} \\
\hline & $\begin{array}{l}\text { Crud OR } \\
\text { (CI) }\end{array}$ & $\begin{array}{l}\text { Adjusted } \\
\text { OR }\end{array}$ & Crud OR & $\begin{array}{l}\text { Adjusted } \\
\text { OR }\end{array}$ & Crud OR & $\begin{array}{l}\text { Adjusted } \\
\text { OR }\end{array}$ & Crud OR & $\begin{array}{l}\text { Adjusted } \\
\text { OR }\end{array}$ \\
\hline \multicolumn{9}{|l|}{ Gender } \\
\hline Female & 1 & & 1 & 1 & 1 & 1 & 1 & 1 \\
\hline Male & $\begin{array}{l}0.49 \\
(0.38- \\
0.64\left(^{c}\right.\end{array}$ & $\begin{array}{l}0.69 \\
(0.52- \\
0.92)^{a}\end{array}$ & $\begin{array}{l}0.31 \\
(0.22- \\
0.43)^{c}\end{array}$ & $\begin{array}{l}0.33 \\
(0.52- \\
0.52)^{c}\end{array}$ & $\begin{array}{l}1.15 \\
(0.75- \\
1.78)\end{array}$ & $\begin{array}{l}1.86 \\
(1.05- \\
3.31)^{a}\end{array}$ & $\begin{array}{l}0.64 \\
(0.50- \\
0.81)^{c}\end{array}$ & $\begin{array}{l}0.66 \\
(0.47- \\
0.91)^{a}\end{array}$ \\
\hline age & $\begin{array}{l}1.02 \\
(1.00- \\
1.03)^{b}\end{array}$ & $\begin{array}{l}1.01(1.00 \\
-1.03)\end{array}$ & $\begin{array}{l}1.02 \\
(1.01- \\
1.04)^{a}\end{array}$ & $\begin{array}{l}1.02 \\
(1.00- \\
1.04)\end{array}$ & $\begin{array}{l}1.05 \\
(1.02- \\
1.07)^{\mathrm{c}}\end{array}$ & $\begin{array}{l}1.04 \\
(1.01- \\
1.07)^{\mathrm{a}}\end{array}$ & $\begin{array}{l}1.08 \\
(1.07- \\
1.09)^{c}\end{array}$ & $\begin{array}{l}1.08 \\
(1.06- \\
1.10)^{c}\end{array}$ \\
\hline \multicolumn{9}{|l|}{$\begin{array}{l}\text { Marital } \\
\text { status }\end{array}$} \\
\hline Married & 1 & 1 & 1 & & 1 & & 1 & \\
\hline Single & $\begin{array}{l}2.10 \\
(1.49- \\
2.96)^{c}\end{array}$ & $\begin{array}{l}1.54 \\
(1.06- \\
2.24)^{a}\end{array}$ & $\begin{array}{l}2.41 \\
(1.63- \\
3.55)^{\mathrm{C}}\end{array}$ & $\begin{array}{l}1.52 \\
(1.00- \\
2.33)^{a}\end{array}$ & $\begin{array}{l}1.74 \\
(0.88- \\
3.41)\end{array}$ & $\begin{array}{l}1.59 \\
(0.76- \\
3.34)\end{array}$ & $\begin{array}{l}1.66 \\
(1.14- \\
2.41)^{b}\end{array}$ & $\begin{array}{l}0.80 \\
(0.52- \\
1.23)\end{array}$ \\
\hline $\begin{array}{l}\text { Education } \\
\text { years }\end{array}$ & $\begin{array}{l}0.94 \\
(0.92- \\
0.96)^{c}\end{array}$ & - & $\begin{array}{l}0.92 \\
(0.89- \\
0.94)^{\mathrm{C}}\end{array}$ & - & $\begin{array}{l}0.97 \\
(0.93- \\
1.01)\end{array}$ & - & $\begin{array}{l}0.91 \\
\left(0.88^{-}\right. \\
0.93)^{\mathrm{C}}\end{array}$ & - \\
\hline \multicolumn{9}{|l|}{$\begin{array}{l}\text { Economic } \\
\text { status }\end{array}$} \\
\hline Poor & 1 & 1 & 1 & 1 & 1 & 1 & 1 & 1 \\
\hline Moderate & $\begin{array}{l}1.66 \\
(1.21- \\
2.27)^{\mathrm{C}}\end{array}$ & $\begin{array}{l}1.62(1.18- \\
2.22)^{b}\end{array}$ & $\begin{array}{l}1.56 \\
(1.08- \\
2.25)^{a}\end{array}$ & $\begin{array}{l}1.43 \\
(0.98- \\
2.08)\end{array}$ & $\begin{array}{l}1.97 \\
(1.10- \\
3.51)^{a}\end{array}$ & $\begin{array}{l}1.95 \\
(1.08- \\
3.53)^{a}\end{array}$ & $\begin{array}{l}1.02 \\
(0.75- \\
1.37)\end{array}$ & $\begin{array}{l}0.82 \\
(0.59- \\
1.13)\end{array}$ \\
\hline Reach & $\begin{array}{l}1.86 \\
(1.37- \\
2.53)^{c}\end{array}$ & $\begin{array}{l}1.60 \\
(1.15- \\
2.20)^{b}\end{array}$ & $\begin{array}{l}1.89 \\
(1.33- \\
2.70)^{c}\end{array}$ & $\begin{array}{l}1.46 \\
(1.00 \\
-2.13)^{a}\end{array}$ & $\begin{array}{l}1.95 \\
(1.09- \\
3.50)^{a}\end{array}$ & $\begin{array}{l}1.71 \\
(0.92- \\
3.17)\end{array}$ & $\begin{array}{l}1.35 \\
(1.02- \\
1.79)^{a}\end{array}$ & $\begin{array}{l}0.81 \\
(0.59- \\
1.12)\end{array}$ \\
\hline BMI & $\begin{array}{l}1.08 \\
(1.05- \\
1.10)^{c}\end{array}$ & $\begin{array}{l}1.06 \\
(1.04- \\
1.09)^{\mathrm{c}}\end{array}$ & $\begin{array}{l}1.08 \\
(1.05- \\
1.11)^{c}\end{array}$ & $\begin{array}{l}1.05 \\
(1.02- \\
1.08)^{\mathrm{C}}\end{array}$ & $\begin{array}{l}1.08 \\
(1.04- \\
1.13)^{\mathrm{C}}\end{array}$ & $\begin{array}{l}1.11 \\
(1.07- \\
1.16)^{\mathrm{C}}\end{array}$ & $\begin{array}{l}1.05 \\
(1.03- \\
1.08)^{\mathrm{C}}\end{array}$ & $\begin{array}{l}1.03 \\
(1.00- \\
1.06)^{a}\end{array}$ \\
\hline \multicolumn{9}{|l|}{$\begin{array}{l}\text { Cigarette } \\
\text { smoking }\end{array}$} \\
\hline No smoker & 1 & - & 1 & 1 & 1 & & 1 & \\
\hline
\end{tabular}

${ }^{1}$ logistic regression, ${ }^{2}$ multinomial logistic regression with true negative self-reported diabetes as reference category

${ }^{a}$ p-value $<0.05,{ }^{b}$ p-value $<0.01,{ }^{c}$ p-value $<0.001$ 


\begin{tabular}{|c|c|c|c|c|c|c|c|c|}
\hline \multirow{2}{*}{$\begin{array}{l}\text { Variables } \\
\text { Ex-smoker }\end{array}$} & \multicolumn{2}{|c|}{$\begin{array}{l}\text { disagreement self- } \\
\text { reported }^{1}\end{array}$} & \multicolumn{2}{|c|}{$\begin{array}{l}\text { false positive self- } \\
\text { reported }^{2}\end{array}$} & \multicolumn{2}{|c|}{$\begin{array}{l}\text { false negative self- } \\
\text { reported }^{2}\end{array}$} & \multicolumn{2}{|c|}{$\begin{array}{l}\text { true positive self- } \\
\text { reported }^{2}\end{array}$} \\
\hline & $\begin{array}{l}0.87 \\
(0.55- \\
1.37)\end{array}$ & - & $\begin{array}{l}0.94 \\
(0.55- \\
1.59)\end{array}$ & $\begin{array}{l}1.40 \\
(0.78- \\
2.50)\end{array}$ & $\begin{array}{l}1.04 \\
(0.44- \\
2.43)\end{array}$ & $\begin{array}{l}0.74 \\
(0.30- \\
1.83)\end{array}$ & $\begin{array}{l}2.45 \\
(1.75- \\
3.41)^{\mathrm{C}}\end{array}$ & $\begin{array}{l}2.12 \\
(1.44- \\
3.14)^{c}\end{array}$ \\
\hline Smoker & $\begin{array}{l}0.88 \\
(0.62- \\
1.24)\end{array}$ & - & $\begin{array}{l}0.65 \\
(0.42- \\
1.02)\end{array}$ & $\begin{array}{l}1.30 \\
(0.76- \\
2.21)\end{array}$ & $\begin{array}{l}1.51 \\
(0.88- \\
2.58)\end{array}$ & $\begin{array}{l}1.46 \\
(0.77- \\
2.76)\end{array}$ & $\begin{array}{l}0.88 \\
(0.62- \\
1.26)\end{array}$ & $\begin{array}{l}1.22 \\
(0.79- \\
1.87)\end{array}$ \\
\hline \multicolumn{9}{|l|}{ Drug use } \\
\hline No & 1 & - & 1 & - & 1 & & 1 & \\
\hline Yes & $\begin{array}{l}0.70 \\
(0.46- \\
1.06)\end{array}$ & - & $\begin{array}{l}0.57 \\
\left(0.33^{-}\right. \\
0.97)^{\mathrm{a}}\end{array}$ & - & $\begin{array}{l}1.02 \\
(0.52- \\
2.00)\end{array}$ & - & $\begin{array}{l}0.85 \\
(0.58- \\
1.25)\end{array}$ & - \\
\hline \multicolumn{9}{|l|}{$\begin{array}{l}\text { Alcohol } \\
\text { use }\end{array}$} \\
\hline No & 1 & - & 1 & 1 & 1 & & 1 & 1 \\
\hline Yes & $\begin{array}{l}0.81 \\
(0.54- \\
1.19)\end{array}$ & - & $\begin{array}{l}0.75 \\
(0.47- \\
1.19)\end{array}$ & $\begin{array}{l}1.41 \\
(0.83- \\
2.41)\end{array}$ & $\begin{array}{l}0.83 \\
(0.41- \\
1.68)\end{array}$ & $\begin{array}{l}0.66 \\
(0.31- \\
1.41)\end{array}$ & $\begin{array}{l}0.56 \\
(0.36- \\
0.88)^{a}\end{array}$ & $\begin{array}{l}0.65 \\
(0.40- \\
1.06)\end{array}$ \\
\hline \multicolumn{9}{|l|}{ HTN } \\
\hline No & 1 & - & 1 & 1 & 1 & & 1 & 1 \\
\hline Yes & $\begin{array}{l}1.36 \\
(1.04- \\
1.78)^{a}\end{array}$ & - & $\begin{array}{l}1.39 \\
(1.00- \\
1.92)^{\mathrm{a}}\end{array}$ & $\begin{array}{l}0.98 \\
(0.68- \\
1.42)\end{array}$ & $\begin{array}{l}2.10 \\
(1.25- \\
3.22)^{b}\end{array}$ & $\begin{array}{l}1.18(0.69- \\
2.02)\end{array}$ & $\begin{array}{l}3.27 \\
(2.56- \\
4.17)^{\mathrm{c}}\end{array}$ & $\begin{array}{l}1.79 \\
(1.35- \\
2.37)^{c}\end{array}$ \\
\hline \multicolumn{9}{|l|}{$\begin{array}{l}\text { Family } \\
\text { history of } \\
\text { DM }\end{array}$} \\
\hline No & 1 & 1 & 1 & 1 & 1 & 1 & 1 & 1 \\
\hline $\begin{array}{l}\text { Second } \\
\text { degree }\end{array}$ & $\begin{array}{l}0.88 \\
(0.57- \\
1.36)\end{array}$ & $\begin{array}{l}0.92 \\
(0.59- \\
1.43)\end{array}$ & $\begin{array}{l}1.28 \\
(0.81- \\
2.05)\end{array}$ & $\begin{array}{l}1.32 \\
(0.82- \\
2.12)\end{array}$ & $\begin{array}{l}0.22 \\
(0.05- \\
0.89)^{a}\end{array}$ & $\begin{array}{l}0.27 \\
(0.07- \\
1.14)\end{array}$ & $\begin{array}{l}1.78 \\
(1.21- \\
2.59)^{b}\end{array}$ & $\begin{array}{l}2.53 \\
(1.69- \\
3.81)^{c}\end{array}$ \\
\hline $\begin{array}{l}\text { First } \\
\text { degree }\end{array}$ & $\begin{array}{l}1.60 \\
(1.24- \\
2.06) \mathrm{c}\end{array}$ & $\begin{array}{l}1.57 \\
(1.21- \\
2.04)^{c}\end{array}$ & $\begin{array}{l}2.03 \\
(1.51- \\
2.73)^{c}\end{array}$ & $\begin{array}{l}1.95 \\
(1.44- \\
2.65)^{c}\end{array}$ & $\begin{array}{l}1.25 \\
(0.78- \\
2.00)\end{array}$ & $\begin{array}{l}1.48 \\
(0.91- \\
2.41)\end{array}$ & $\begin{array}{l}2.76 \\
(2.14- \\
3.56)^{c}\end{array}$ & $\begin{array}{l}3.43 \\
(2.61- \\
4.51)^{c}\end{array}$ \\
\hline \multicolumn{9}{|c|}{$\begin{array}{l}{ }^{1} \text { logistic regression, }{ }^{2} \text { multinomial logistic regression with true negative self-reported diabetes as reference } \\
\text { category }\end{array}$} \\
\hline a $p$-value $<c$ & $5,{ }^{b} \mathrm{p}-\mathrm{val}$ & $0.01,^{c}$ & $e<0.00$ & & & & & \\
\hline
\end{tabular}

\section{Discussion}


In this study on the validity of self-reported diabetes in a large Kurdish population, we found self-reported diabetes had a moderate sensitivity of $78.5 \%$, a high specificity of $93.9 \%$, a fairly good positive predictive value for selfreporting diabetes of $58.7 \%$, and a high negative predictive value for self-reporting no diabetes of $98.0 \%$. The agreement between self-reported diabetes and reference criteria was fairly good with Kappa of $65.1 \%$ and concordance of $92.3 \%$. Besides, we showed that the demographic, anthropometric, and habitual features of subjects had largely influenced the accuracy of self-reported diabetes. In this case, being female, increase in age, increase in BMI, being an ex-smoker, having HTN, and family history of diabetes increase the odds of true positive rate in diabetes self-reports. We found $31 \%$ of diabetic participants (120 out of a total of 386) were not under any medication for diabetes. The previous reports on this issue showed almost the same statistics [15, 16]; however, we demonstrated an updated validation of diabetes self-reports among a large Kurdish population of Iran.

The epidemiological surveys commonly applied either self-report or medical records of chronic diseases to estimate their incidence or prevalence [17]. Among chronic diseases, self-reports of diabetes were identified to be more accurate with a higher level of agreement [18-20]. Our findings on the accuracy of self-reported diabetes were in line with the recent similar studies that showed the sensitivity of 75-79.3\% and specificity of $95.8-98.4 \%$ [16, 21]. However, older previous studies showed lower sensitivity of $61.5-69.7 \%$ for diabetes self-reports [20, 22]. This increasing trend in the accuracy of diabetes self-reports can be explained by the increase in awareness of society and the development of the health care system over time [23]. Meanwhile, the difference in this accuracy over time can be due to the different demographic features of the studied population since we similar to previous studies revealed that the accuracy of diabetes self-reports was largely dependent on the baseline characteristics of study participants $[4,16,20]$.

The results of the multivariable analysis showed that women were more likely to have a disagreement of selfreported diabetes with the reference, higher false positive and true positive rates and lower false negative rate than men. One explanation for this finding is that women take better self-care behaviors and use more health care services [24]. Moreover, women take more attention to their dietary consumption. In this instance, they tend to count daily carbohydrates intake and consume less fat [25]. Thus, they were more likely to find themselves in diabetic condition and reported more true positives and false positives. We also found that increment in the age of study participants was associated with higher odds of true positive and false negative rates of self-reported diabetes. The higher false negative self-reports in older participants can be due to a recall bias because of Alzheimer's disease or age-related memory loss [26] and higher true positive self-reports among older individuals can be due to more health care delivery and more opportunity to undergo blood sugar testing in this population [24]. We also observed that increment in BMI was associated with higher odds of the discordance between diabetes self-reports and the reference criteria, higher true positives, false negatives, and false positives of self-reported diabetes. In the previous studies in line with this study, obesity, as well as an increase in BMI, resulted in higher odds of diabetes development in this population and consequently higher true positive and false negative rates [16, $20,27]$. This finding can be attributed to insulin resistance condition in obesity as well as poor self-care of overweight and obese individuals $[28,29]$.

In this study, participants with HTN were more likely to truly report their diabetes. This finding can be due to better monitoring of other metabolic syndrome risk factors in this population and higher awareness about their health. In line with previous studies, we observed no significant change in the odds of false negative and false positive rates among populations with HTN [16]. 
Positive family history of diabetes, particularly from the first-degree relatives, showed a high level of discordance in diabetes self-reports. In this instance, subjects with positive family history were more likely to develop diabetes that this issue explained higher true positive rates of diabetes self-reports in this group. Besides, similar to previous studies, subjects with positive family history tend to report diabetes more frequently which leads to higher false positive rates $[16,30]$.

\section{Strengths And Limitations}

This study had several strengths worth to be stated. This study was a large population-based survey derived from the PERSIAN cohort of Iran and had a low risk of attrition bias with a high response rate (91\%) of enrolled residents of Dehgolan, the Kurdish region of Iran. Thus, we could generalize our findings to the whole Kurdish population of Iran. This study also had several limitations worth to be discussed. This study examined the self-reported prevalent diabetes; thus, this validation could not be applied for the studies investigating incident diabetes. As stated, this validation was conducted in the west part of Iran and due to the racial, ethnic, and socio-cultural diversity of other regions of Iran, we required further validation to determine the accuracy of self-reported diabetes in other parts of Iran and to elucidate the impact of socio-cultural nature of each region on the accuracy and discordance of selfreported diabetes.

\section{Conclusion}

We have found self-reported diabetes with moderate sensitivity indicating high awareness of the general Kurdish population of Iran about their diabetic status, high specificity, fairly good PPV, and very high NPV, reflecting good accuracy of self-reported diabetes for detecting diabetes in this population. We also found good agreement between self-reported diabetes and reference criteria. Thus, diabetes self-reporting could be used as a valid tool to identify diabetes prevalence in future epidemiological studies on the Kurdish population of Iran. Besides, we revealed sociodemographic and habitual characteristics of individuals have largely affected this validity and should be considered to warrant more accurate estimation.

\section{Abbreviations}

Se: Sensitivity

Sp: Specificity

NPV: Negative Predict Value

PPV: Positive Predict Value

HTN: Hypertension

BMI: Body Mass Index

PRC: Precision-Recall Curve

FP: False Positive

TP: True Positive 
FN: False Negative

TN: True Negative

FBS: Fasting Blood Sugar

DehPCS: The Dehgolan Prospective Cohort Study

PERSIAN: Prospective Epidemiological Cohort Studies in Iran

\section{Declarations}

\section{Acknowledgements}

We thank all midwives, doctors, and laboratory workers at the Dehgolan Prospective Cohort study for assisting in the study.

\section{Author contributions}

FM, NP, MFK and YM organized data acquisition, collected the data, participated in the study design, and wrote the manuscript. FM and YM analyzed the data and reviewed critically the manuscript. YM, GHM and HD evaluated and revised the manuscript.

\section{Funding}

This research did not receive any specific grant from funding agencies in the public, commercial, or not-for-profit sectors.

\section{Availability of data and material}

The data of this study are available on request from the corresponding author (FM). The data are not publicly available due to privacy and ethical restrictions.

\section{Conflict of interest}

The authors have no financial, personal, political, intellectual or religious interests to declare.

\section{Ethical approval}

The study was approved by the Ethics Committee of the Kurdistan University of Medical Sciences, Sanandaj, Iran. (no. IR.MUK.REC.1400.150).

\section{Consent for Publication}

Not applicable.

\section{Competing Interests}

The authors declare that they have no competing interests. 


\section{References}

1. Goto, A., et al., Validity of diabetes self-reports in the Saku diabetes study. Journal of epidemiology, 2013: p. JE20120221.

2. Atlas, D., International diabetes federation. IDF Diabetes Atlas, 7th edn. Brussels, Belgium: International Diabetes Federation, 2015.

3. Organization, W.H., Web World Health Organization-Diabetes country profiles. 2016, Diakses.

4. Yuan, X., et al., Validity of self-reported diabetes among middle-aged and older Chinese adults: the China Health and Retirement Longitudinal Study. BMJ open, 2015. 5(4): p. e006633.

5. Pinheiro, P.C., et al., Differences between self-reported and laboratory measures of diabetes, chronic kidney disease, and hypercholesterolemia. Ciência \& Saúde Coletiva, 2021. 26: p. 1207-1219.

6. Danaei, G., et al., Diabetes prevalence and diagnosis in US states: analysis of health surveys. Population health metrics, 2009. 7(1): p. 1-13.

7. Schneider, A.L., et al., Validity and reliability of self-reported diabetes in the atherosclerosis risk in communities study. American journal of epidemiology, 2012. 176(8): p. 738-743.

8. Espelt, A., et al., Validity of self-reported diabetes in health interview surveys for measuring social inequalities in the prevalence of diabetes. J Epidemiol Community Health, 2012. 66(7): p. e15-e15.

9. Najafi, F., et al., The reliability of self-reporting chronic diseases: how reliable is the result of population-based cohort studies. Journal of preventive medicine and hygiene, 2019. 60(4): p. E349.

10. Malta, D.C., et al., Prevalence of diabetes mellitus as determined by glycated hemoglobin in the Brazilian adult population, National Health Survey. Revista Brasileira De Epidemiologia, 2019. 22.

11. Vellakkal, S., et al., Socioeconomic inequalities in non-communicable diseases prevalence in India: disparities between self-reported diagnoses and standardized measures. PloS one, 2013. 8(7): p. e68219.

12. Ghorbani, Z., Validating self-report of diabetes and hypertension use by participants in the pilot phase of Azar cohort study: comparison with paraclinic indicators $₫$ medical history and medical examination. 2018, Tabriz University of Medical Sciences, Faculty of Nutrition and Food Sciences.

13. Poustchi, H., et al., Prospective Epidemiological Research Studies in Iran (the PERSIAN Cohort Study): Rationale, Objectives, and Design. Am J Epidemiol, 2018. 187(4): p. 647-655.

14. Moradpour, F., et al., Cohort profile: Dehgolan prospective cohort study (DehPCS) on non-communicable diseases in a Kurdish community in the west of Iran. Epidemiol Health, 2021: p. e2021075.

15. Tanjani, P.T., et al., The prevalence of diabetes mellitus (DM) type II among Iranian elderly population and its association with other age-related diseases, 2012. Archives of gerontology and geriatrics, 2015. 60(3): p. 373379.

16. Moradinazar, M., et al., Validity of self-reported diabetes varies with sociodemographic charecteristics: Example from Iran. Clinical Epidemiology and Global Health, 2020. 8(1): p. 70-75.

17. Okura, Y., et al., Agreement between self-report questionnaires and medical record data was substantial for diabetes, hypertension, myocardial infarction and stroke but not for heart failure. Journal of clinical epidemiology, 2004. 57(10): p. 1096-1103.

18. Fontanelli, M.d.M., et al., Validation of self-reported diabetes in a representative sample of São Paulo city. Revista de Saúde Pública, 2017. 51. 
19. Wu, S.-C., C. Li, and D. Ke, The agreement between self-reporting and clinical diagnosis for selected medical conditions among the elderly in Taiwan. Public health, 2000. 114(2): p. 137-142.

20. Huerta, J.M., et al., Accuracy of self-reported diabetes, hypertension, and hyperlipidemia in the adult Spanish population. DINO study findings. Revista Española de Cardiología (English Edition), 2009. 62(2): p. $143-152$.

21. Chun, H., I.-H. Kim, and K.-D. Min, Accuracy of self-reported hypertension, diabetes, and hypercholesterolemia: analysis of a representative sample of Korean older adults. Osong public health and research perspectives, 2016. 7(2): p. 108-115.

22. Golozar, A., et al., Diabetes mellitus and its correlates in an Iranian adult population. PloS one, 2011. 6(10): p. e26725.

23. Desai, D., et al., Health care utilization and burden of diabetic ketoacidosis in the US over the past decade: a nationwide analysis. Diabetes care, 2018. 41(8): p. 1631-1638.

24. Keene, J. and X. Li, Age and gender differences in health service utilization. Journal of Public Health, 2005. 27(1): p. 74-79.

25. Chang, S.H., Y.Y. Chang, and L.Y. Wu, Gender differences in lifestyle and risk factors of metabolic syndrome: Do women have better health habits than men? Journal of clinical nursing, 2019. 28(11-12): p. 2225-2234.

26. Kumar, A., et al., Alzheimer disease. 2018.

27. Wilder, R.P., et al., Socio-economic status and undiagnosed diabetes. Diabetes research and clinical practice, 2005. 70(1): p. 26-30.

28. Tee, J.Y.H., et al., Obesity and unhealthy lifestyle associated with poor executive function among Malaysian adolescents. PLoS One, 2018. 13(4): p. e0195934.

29. Schnurr, T.M., et al., Obesity, unfavourable lifestyle and genetic risk of type 2 diabetes: A case-cohort study. Diabetologia, 2020. 63(7): p. 1324-1332.

30. Soriguer, F., et al., Prevalence of diabetes mellitus and impaired glucose regulation in Spain: the Di@ bet. es Study. Diabetologia, 2012. 55(1): p. 88-93.

\section{Figures}




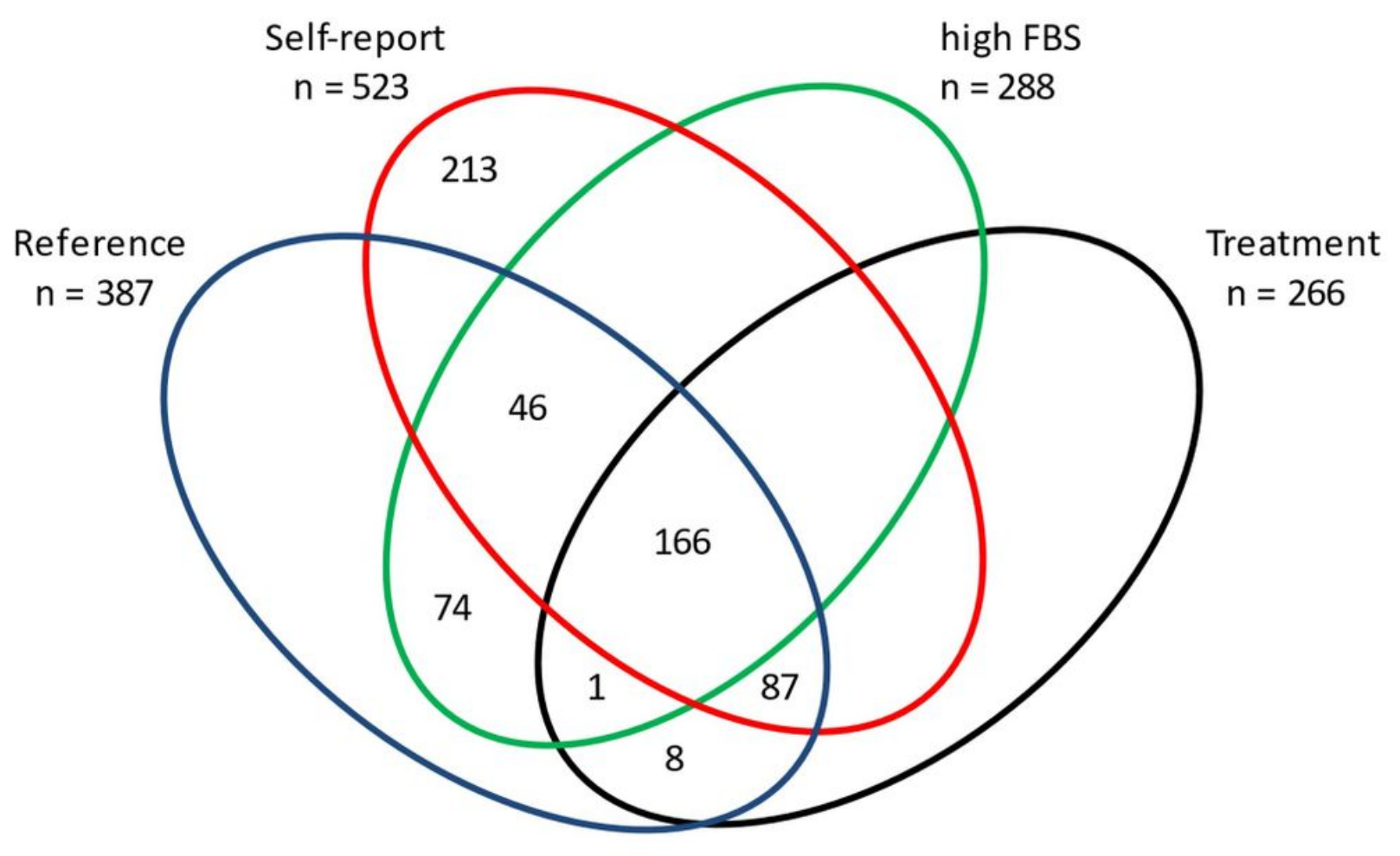

Figure 1

frequency overlap of self-reported diabetes and diabetes measured by reference criteria (high FBS + treatment) 


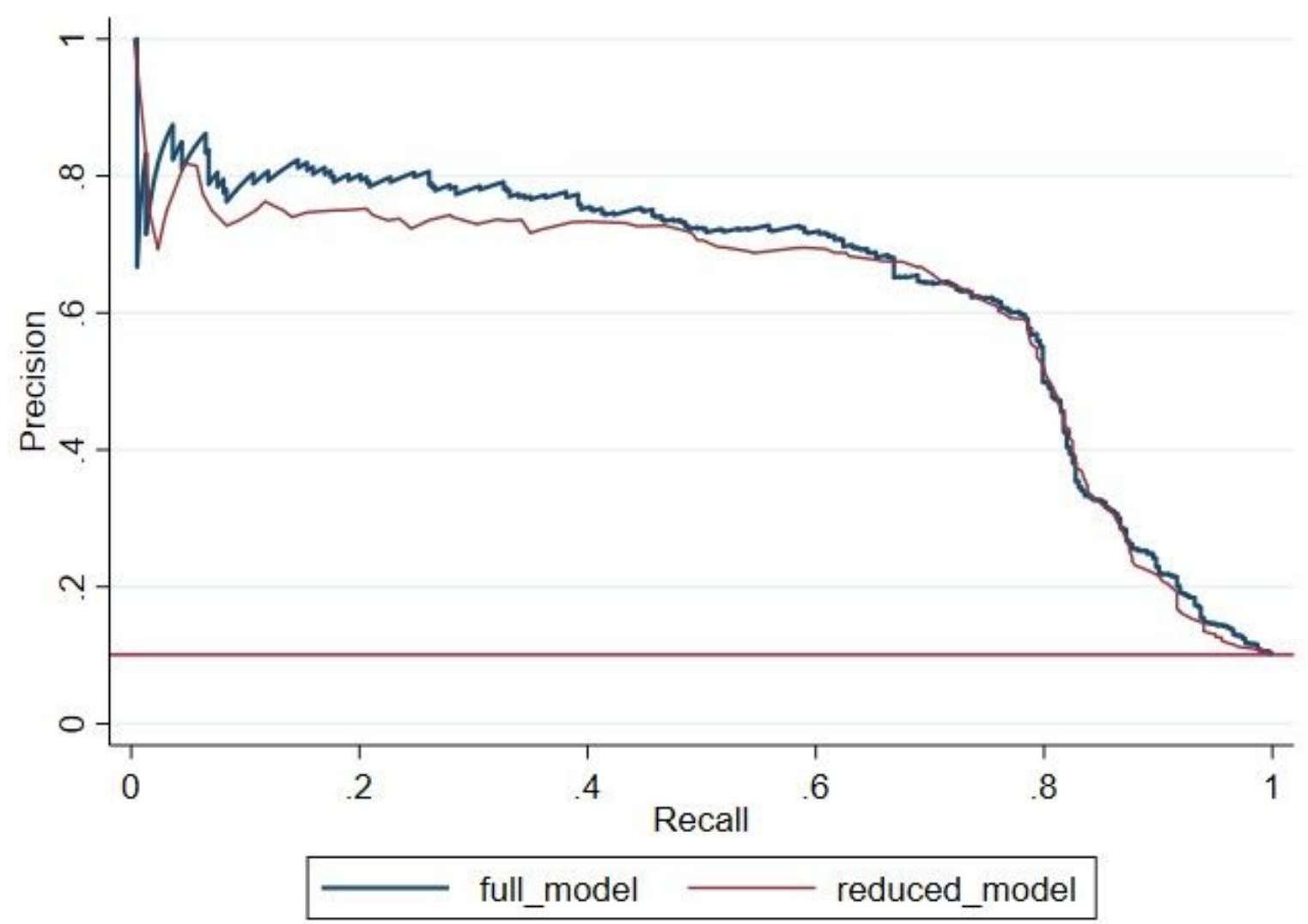

Figure 2

diagnostic characteristics of reduced model (Self-reported diabetes, Sex, Age) in comparison with full model (Selfreported diabetes, sex, age, marital status, economic status, BMI, smoking status, alcohol use, HTN, and family history of HTN) 